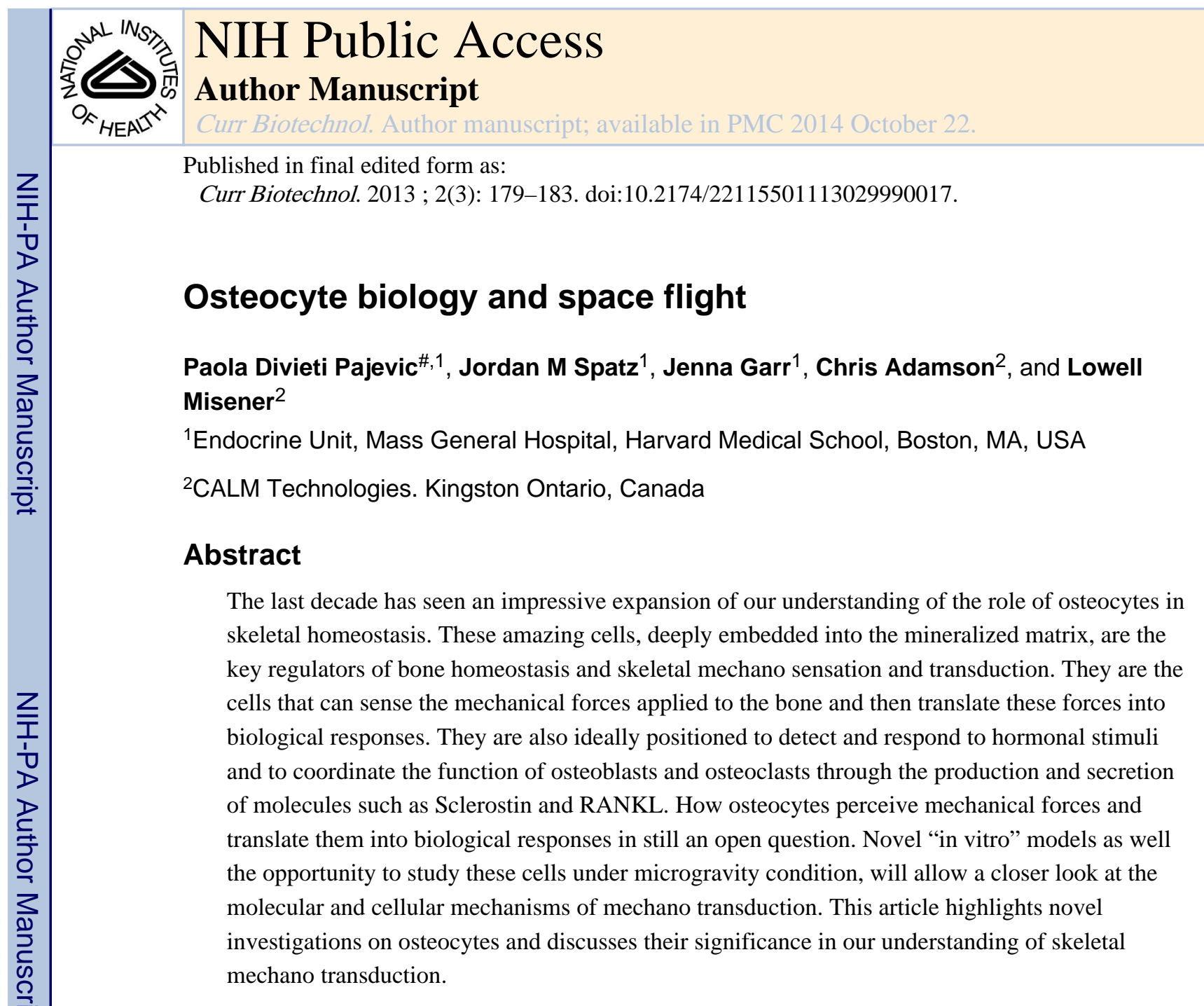

\title{
Introduction
}

The desire to understand and explore the universe has been in the mind and heart of men for centuries. The dream of exploring the universe became a reality in 1957 when a stray dog named Laika, was sent in space onboard a Russian vehicle. Although she did not survive the mission, Laika paved the ground for human space exploration that culminated in 1969 with the successful landing of astronauts Armstrong and Aldrin on the Moon. From these early flights it was clear that the lack of gravity, associated with exposure to radiation had a toll on the human body. As humans began spending longer periods of time in space, it became evident that weightlessness affected the health of bones, muscles, the cardiovascular and immune systems. The organ that is most affected by reduced gravity is the skeleton, and Galileo was indeed the first to observe and describe the consequences of loading (or lack of it) on the skeleton. Indeed mechanical loading is required for proper development and maintenance of the musculoskeletal system. It has been recognized for over a century that loading is fundamental for bone health, and that reduced loading (as in prolonged bed rest or space flight) is invariably associated with bone loss. In 1892, Wolff theorized (Wolff's law on bone transformation)(1) that mechanical stress is the driving force for the architecture of

\footnotetext{
\#Corresponding author: Paola Divieti Pajevic, MD PhD, Harvard Medical School, Massachusetts General Hospital, Endocrine Unit, Thier 1101, Boston, MA 02114, ppajevic@partners.org, 617-726-6184.
} 
bone. The central concept is that bone adapts its form to mechanical demands (bone modeling). Bone resorption, driven by osteoclasts, and bone formation, osteoblastsmediated, occur on different surfaces of the skeleton such as the bone undergoes reshaping to adapt to different loading conditions. Bone remodeling, on the other hand, is the continuous and spatially coupled, resorption and formation of the skeleton to preserve functional integrity. In the late 80's, Frost speculated the existence of a "mechanostat" of bone that distinguishes between bone modeling (shape change) and remodeling (replacement only) (2). Example of bone adaptation to loading is evident in professional athletes, where the high load applied to the skeleton results in stronger and bigger bones, whereas in astronauts or in paralyzed patients there is severe bone loss due to unloading.

How the skeleton perceives mechanical forces, and distinguishes between the cellular and molecular mechanisms of mechano-transduction is still under intense investigation. It has been demonstrated that osteocytes, the cells deeply embedded in the bone are the cells capable of sensing mechanical forces and transforming these forces into biological responses. This article will review recent progress in understanding osteocyte mechanosensation and how experiments on the International Space Station might provide unique cues on the functions of these cells.

\section{Osteocytes, mechano-sensation and mechano-transduction}

How does the skeleton perceive mechanical forces? Mechanotransduction, i.e." (the) process of converting physical forces into biochemical signals and integrating these signals into (a) cellular response" is the prerequisite for a functional and healthy skeleton (3). Because mechanical stimuli regulate various cellular functions, including gene expression, protein synthesis, as well as cell proliferation and differentiation, understanding mechanotransduction at the cellular level is key to understanding basic bone biology and designing new treatments for bone diseases such as osteoporosis $(4,5)$.

Osteocytes, the bone cells deeply embedded in the mineralized matrix are thought to be the mechanosensor of bone. Their location, deep within the mineralized matrix and their structural organization of a cellular network, make them ideal to sense mechanical stimuli and to transfer them to the surrounding cells. They derive from osteoblasts that, during the process of bone formation, assume a more differentiated morphology and become entrapped in the matrix that they are actively synthesizing (6-8). Three types of mechanical stimuli have been proposed: (a) direct deformation of the cells via bending or compression of the matrix to which they are attached, (b) fluid flow-induced shear stress and (c) electric fields resulting from electrokinetic effects linked to fluid. The leading theory is that shear stress induced by fluid flow in the lacuno-canalicular system, is indeed the mechanical signal perceived by the cells.

The most convincing evidence that osteocytes are indeed the mechanosensors of bone, comes from a work of Tatsumi et al. (9) who engineered a mouse model in which osteocytes could be selectively ablated upon diphtheria toxin administration. Using this model, the authors demonstrated not only that osteocytes are key regulators of skeletal homeostasis 
(these mice have severe osteopenia), but that they are resistant to unloading induced bone loss, as achieved by tail-suspension (9).

Osteocytes respond to mechanical forces (or lack of them) by secreting several molecules such as nitric oxide (NO), prostaglandin $\mathrm{E}_{2}\left(\mathrm{PEG}_{2}\right)$ and Sclerostin (6), to name a few. Sclerostin, the product of the SOST gene, is an osteocyte-specific protein and recently has emerged as an important therapeutic target for bone diseases such as osteoporosis and osteopenia. This osteocyte-specific protein inhibits bone formation, both in vitro and in vivo, by directly reducing proliferation and differentiation of osteoblasts via the canonical Wnt signaling pathway. It has been shown that Sclerostin acts by binding the low-density lipoprotein receptor 5 and 6 (LRP5 and 6) and inhibit Wnt- $\beta$ catenin signaling pathway and that both the mRNA and the protein are regulated by mechanical forces. Recent studies in both animal models and patients have shown that Sclerostin synthesis and expression is highly regulated by mechanical forces. Several studies have shown that the protein is elevated during reduced loading as achieved by bed rest $(10,11)$, paralysis $(11)$ or hind-limb unloading (12) and it is reduced during increased loading (12).

\section{Osteocytes and microgravity}

What are the effects of microgravity (or reduced loading) on osteocytes? Research preformed by Krempein et al. (13) first demostrated the effects of reduced mechanical loading on osteocyte morphology. Rats were immobilized by spinal cord severing, plaster cast, or nerve dissection. Three weeks of immobilzation caused a significant decrease percentage of small metabolitcally inactive osteocytes (spinal cord severing, $-20 \%$, plaster cast, $-15.4 \%$ ) and a corresponding increase percentatge of mature enlarged osteocytes (spinal cord severing, $+12.6 \%,+14.6 \%$ ). These morphology observations support the theory of immobolization leading to an increased number of mature osteocytes secreting sclerostin, thereby inhibiting bone formation. In addition, an increase percentage of empty lacunae was observed supporting the theory of immobilized induced osteocyte apoptosis as a initiator of osteoclast activity (14-16).

Twenty five years after the Krempein study, a Russian "Bion-11" biosatellite launched (2001) carrying two Macaca mullatta monkeys for a fourteen day mission. Examination of iliac crest biopsies of the flight animals showed that mature osteocytes had increased specific volume of the Golgi complex and increased osteolytic activity (17). However, a definitive correlation between immobilized morphological changes and underlying gene/ protein expression changes currently cannot be established as both the Krempein et al. and Russian flights occurred prior to the discovery of osteocyte specific genes over the past several years.

One of the limitations of osteocyte biology is the paucity of available cell lines that are suitable for studing the intricated and complicated mechanisms of mechano-tranduction. Currently the osteocytic cell lines availabe are less than a handfull. The most studied and characterized cell line is MLOY-4, (18) a conditionally immortalized cell line derived from long bone of mice in which the SV40 antigen was driven by the osteocalcin promoter. Although these cells possess most of the characteristic of an osteocyte, they do not express 
high level of Sost/sclerostin or express another osteocyte specific gene, namely Fibroblast Growth Factor-23 (FGF23) $(19,20)$. Other osteocytic cell lines currently available are Oc14 (21) and two novel lines derived from condionally immortalized animals expressing GFP under the Dentin Matrix protein-1 (DMP-1) promoter (20,22). These novel cells more faithfull recapitulate the characteristic of "in vivo" osteocytes and will provide an unavulabe new tool to further understand osteocyte biology.

\section{Flight Hardware for Bone Biology Studies}

One of the challenges in studying "in vitro" models in microgravity is the constrained logistic of the environment. At present, few payloads have flown successfully on board of spacecrafts and few are currently available on the International Space Station (ISS). One of the payloads developed and used for bone cell culture is OSTEO (Fig.1). The OSTEO system was originally developed by the Canadian Space Agency (CSA) as a cell culture rack operated by astronauts. It was designed for the shuttle, and flew on several missions, including STS-95 and STS-107 (no data was collected from STS-107). During the Columbia investigation and return to flight timeframe, OSTEO was upgraded to a fully automated payload, eOSTEO. A Russian FOTON biosatellite in 2007 flew with two eOSTEO systems aboard; one for CSA experiments and one for European Space Agency (ESA) experiments. Investigations on the Canadian side included effects of parathyroid hormone (PTH)-related peptide (PTHrP) on osteobasts, the CD200 ligand pathway in osteoblasts and osteoclasts, and the cytoskeleton of bone cells in microgravity. European investigators looked at fibrogenesis of osteoblasts, osteoclast differentiation, and the effects of microgravity on the cross-influence of osteoclasts and osteoblasts (23). The eOSTEO system includes automated hardware specifically designed for bone cell cultures and is currently the system of choice for culturing bone and bone derived cells. Over the next couple of years eOSTEO will flew to the ISS carrying a novel osteocytic cell line, recently developed in our laboratory as part of an NIH/NASA funded project. Results from this experiment will provide novel insight into osteocyte mechanisms of mechano-transduction.

Another fully automated payload recently used to investigate the effect of microgravity on bone marrow derived mesenchymal stem cells (BMSC), is a bioreactor from Kayser-Italia (24). The spaceflight bioreactor is comprised of five cylindrical plunger compartments in which cells are cultured on porous ceramic disks (Skelite) and maintained in culture chambers (24). The latest NASA payload for cell culture is the Cell Bio Tech Demo (CBTD), a precursor to new hardware currently developed by the agency and scheduled for delivery to the ISS in 2014. This new hardware, known as the Bioculture System, "will enable long duration cell biology research in space and give scientists a greater understanding of how cells and tissues grow in the absence of gravity".

These payloads operate as a close system that, opposite to a standard "in vitro" culture, is not exposed to a controlled carbon dioxide $\left(\mathrm{CO}_{2}\right)$ concentration (typically $\left.5 \% \mathrm{CO}_{2}\right)$. This setting does not allow free exchange of oxygen and $\mathrm{CO}_{2}$ and requires either the use of specialized culture medium or the addition of buffers to maintain the $\mathrm{pH}$ of the cell cultures within the physiological range. 
Routine 2D culture of bone cells might not completely reflect the physiological relationship and forces that bone cells are subjected to, therefore the use of 3D structures, or scaffolds, is preferred when studying osteocytes (or other bone cells) under altered mechanical conditions (loading or microgravity). A multitude of scaffolds of inert support are currently available for bone research. They include collagen-based sponges, hydroxyapatite substrates, and synthetic materials, such as polypropylene (Fig.2). The choice of scaffold is often guided by both the experimental end-point (compatibility of the substrate with the end applications) and the culture conditions (geometry of the scaffolds).

In summary, the field of space biology is just at the beginning and with the completion of the ISS and the utilization of the ISS National Laboratory has open the doors to new avenues of microgravity research. In the near future, with the commercial delivery options (SpaceX Dragon, Orbital Sciences Cygnus) becoming available, opportunities to conduct experiments on board the ISS will become logistically more feasible (although still financially expensive). In time, more investigators will have the opportunity to directly study the effects of microgravity on bone cells and derive from these experimentations new clues on the cellular and molecular mechanisms that govern fundamental bone biology. Only time and continued scientific exploration in space and here on Earth will tell if these future space endeavors will be successful and provide novel therapeutics for the millions of people on Earth, afflicted by bone diseases. We can postulate that a better understanding of the molecular mechanisms of osteocyte mechano sensation and transduction, will generate novel therapeutics that target (or mimic) the stimuli or the cellular responses, rather than its effectors (osteoblasts and osteoclasts) (Fig.3). If the future of space exploration is a human mission to Mars, effective countermeasures must be developed and implemented to prevent dramatic bone loss in astronauts. As shown in Fig.3, there are several levels for intervention: we can simulate mechanical forces; we can simulate cellular mechano sensation or transduction or we can intervene on distal effectors (namely osteocytes products that control osteoblast and osteoclast functions). Current approaches to treat bone disease, such as osteoporosis, regulate the function of distal effectors by blocking osteoclast functions (antiresoprtive agents such as biphosphonate) or enhancing osteoblast activities (anabolic agents, such as teriparatide). New therapies are needed that target osteocytes directly but before this can happen we need a deep understanding of the complexes molecular and cellular mechanism that govern these amazing cells.

\section{Acknowledgments}

This work was supported by NIH grant UH3-AR059655 (PDP)

\section{References}

1. Wolff J. Das Gesetz der Transformation der Knochen Kirschwald. 1892

2. Frost HM. Bone "mass" and the "mechanostat": a proposal. Anat rec. 1987; 219:1-9. [PubMed: 3688455]

3. Iqbal J, Zaidi M. Molecular regulation of mechanotransduction. Biochem Biophys Res Commun. 2005; 328(3):751-755. [PubMed: 15694410]

4. Knothe Tate ML. "Whither flows the fluid in bone?" An osteocyte's perspective. J Biomech. 2003; 36(10):1409-24. [PubMed: 14499290] 
5. Hughes-Fulford M. Signal transduction and mechanical stress. Sci STKE. 2004; 2004(249):RE12. [PubMed: 15353762]

6. Knothe Tate ML, Adamson JR, Tami AE, Bauer TW. The osteocyte. Int J Biochem Cell Biol. 2004; 36(1):1-8. [PubMed: 14592527]

7. Noble BS. The osteocyte lineage. Archives of Biochemistry and Biophysics. 2008; 473(2):106-111. [PubMed: 18424256]

8. Nguyen AM, Jacobs CR. Emerging role of primary cilia as mechanosensors in osteocytes. Bone.

9. Tatsumi S, Ishii K, Amizuka N, Li M, Kobayashi T, Kohno K, Ito M, Takeshita S, Ikeda K. Targeted ablation of osteocytes induces osteoporosis with defective mechanotransduction. Cell Metab. 2007; 5(6):464-475. [PubMed: 17550781]

10. Spatz JM, Fields EE, Yu EW, Pajevic PD, Bouxsein ML, Sibonga JD, Zwart SR, Smith SM. Serum Sclerostin Increases in Healthy Adult Men during Bed Rest. J Clin Endocrinol Metab. 97(9):E1736-E1740. [PubMed: 22767636]

11. Battaglino RA, Sudhakar S, Lazzari AA, Garshick E, Zafonte R, Morse LR. Circulating sclerostin is elevated in short-term and reduced in long-term SCI. Bone. 51(3):600-605. PMCID: PMC3412894. [PubMed: 22575440]

12. Robling AG, Niziolek PJ, Baldridge L, KW C, MR A, I A, SM M, J G-H, TM B, SE H, CH T. Mechanical stimulation of bone in vivo reduces osteocyte expression of Sost/sclerosti. J Biol Chem. 2008; 283(9):5866-5875. [PubMed: 18089564]

13. Krempien B, Manegold C, Ritz E, Bommer J. The influence of immobilization on osteocyte morphology: osteocyte differential count and electron microscopical studies. Virchows Arch A Pathol Anat Histol. 1976; 370(1):55-68. [PubMed: 818789]

14. Aguirre JI, Plotkin LI, Stewart SA, Weinstein RS, Parfitt AM, Manolagas SC, Bellido T. Osteocyte apoptosis is induced by weightlessness in mice and precedes osteoclast recruitment and bone loss. J Bone Miner Res. 2006; 21(4):605-615. [PubMed: 16598381]

15. Cardoso L, Herman BC, Verborgt O, Laudier D, Majeska RJ, Schaffler MB. Osteocyte Apoptosis Controls Activation of Intracortical Resorption in Response to Bone Fatigue. J Bone Miner Res. 2008

16. Plotkin LI, Mathov I, Aguirre JI, Parfitt AM, Manolagas SC, Bellido T. Mechanical Stimulation Prevents Osteocyte Apoptosis: Requirement of Integrins, Src Kinases and Erks. Am J Physiol Cell Physiol. 2005

17. Rodionova NV, Oganov VS, Zolotova NV. Ultrastructural Changes in osteocytes in microgravity conditions. Adv Space Res. 2002; 30(4):765-770. [PubMed: 12528727]

18. Kato Y, Windle JJ, Koop BA, Mundy GR, Bonewald LF. Establishment of an osteocyte-like cell line, MLO-Y4. J Bone Miner Res. 1997; 12(12):2014-2023. [PubMed: 9421234]

19. Liu S, Zhou J, Tang W, Jiang X, Rowe DW, Quarles LD. Pathogenic role of Fgf23 in Hyp mice. Am J Physiol Endocrinol Metab. 2006; 291(1):E38-E49. [PubMed: 16449303]

20. Woo SM, Rosser J, Dusevich V, Kalajzic I, Bonewald LF. Cell line IDG-SW3 replicates osteoblast-to-late-osteocyte differentiation in vitro and accelerates bone formation in vivo. J Bone Miner Res. 26(11):2634-2646. PMCID: PMC3192242. [PubMed: 21735478]

21. Divieti P, Inomata N, Chapin K, Singh R, Juppner H, Bringhurst FR. Receptors for the carboxylterminal region of pth(1-84) are highly expressed in osteocytic cells. Endocrinology. 2001; 142(2):916-925. [PubMed: 11159865]

22. Zhu J, Siclari VA, Liu F, Spatz JM, Chandra A, Divieti Pajevic P, Qin L. Amphiregulin-EGFR signaling mediates the migration of bone marrow mesenchymal progenitors toward PTHstimulated osteoblasts and osteocytes. PLoS One. 7(12):e50099. PMCID: PMC3534030. [PubMed: 23300521]

23. Tamma R, Colaianni G, Camerino C, Di Benedetto A, Greco G, Strippoli M, Vergari R, Grano A, Mancini L, Mori G, Colucci S, Grano M, Zallone A. Microgravity during spaceflight directly affets in vitro osteoclastogenesis and bone resorption. Faseb J. 2009; 23(8):2549-2554. [PubMed: 19329761]

24. Monticone M, Liu Y, Pujic N, Cancedda R. Activation of nervous system development genes in bone marrow derived mesenchymal stem cells following spaceflight exposure. J Cell Biochem. 111(2):442-452. [PubMed: 20658479] 


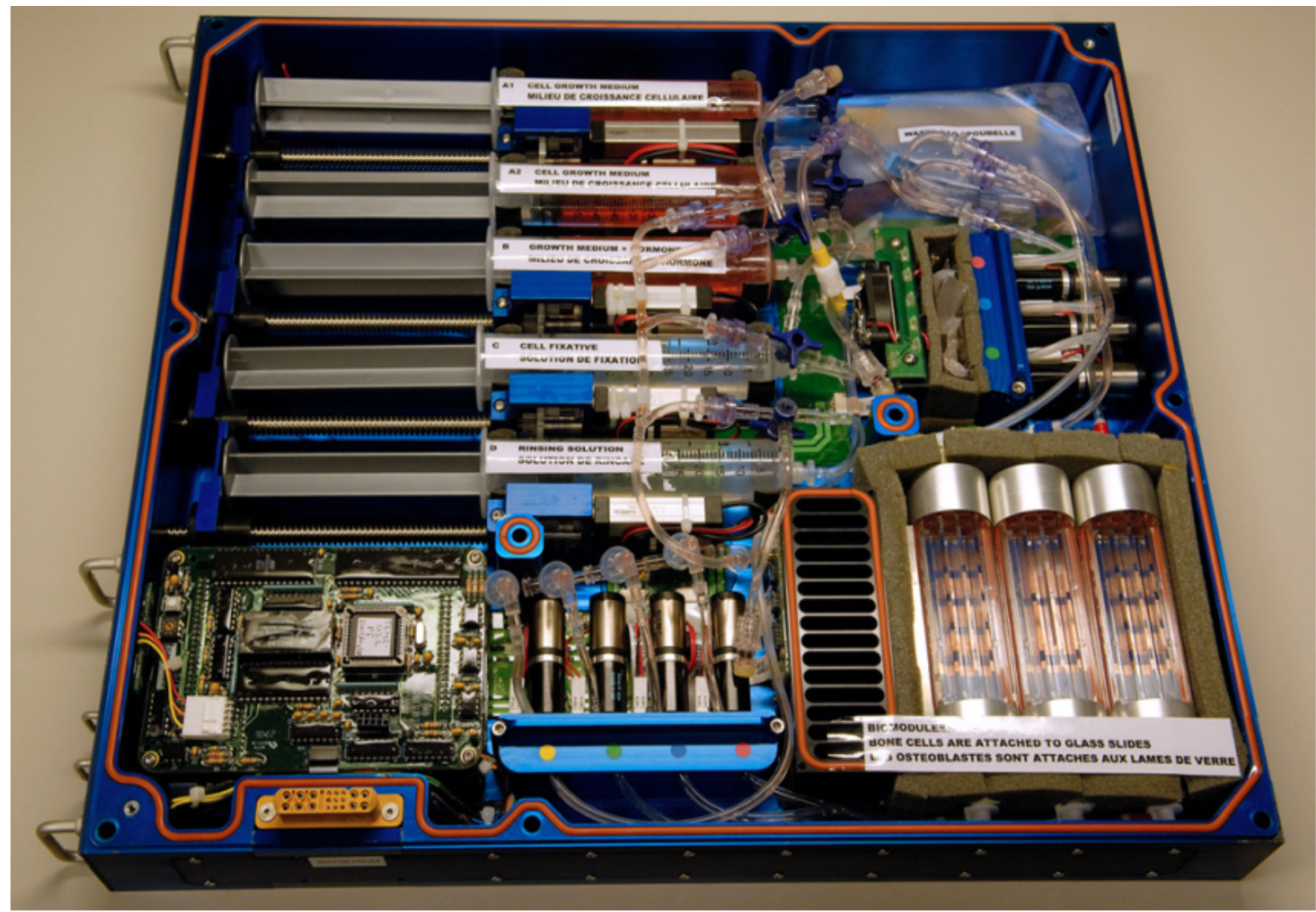

Figure 1.

Osteo/eOsteo flight Hardware. The eOsteo consist of three independent trays each operating at a defined temperature. Each tray houses a closed fluid pathway network, where three cell culture container (bioreactors, bottom right) receive nutrients or other fluids, from any of five syringe reservoirs via a multiple valve system. Hardware courtesy of the Canadian Space Agency. 


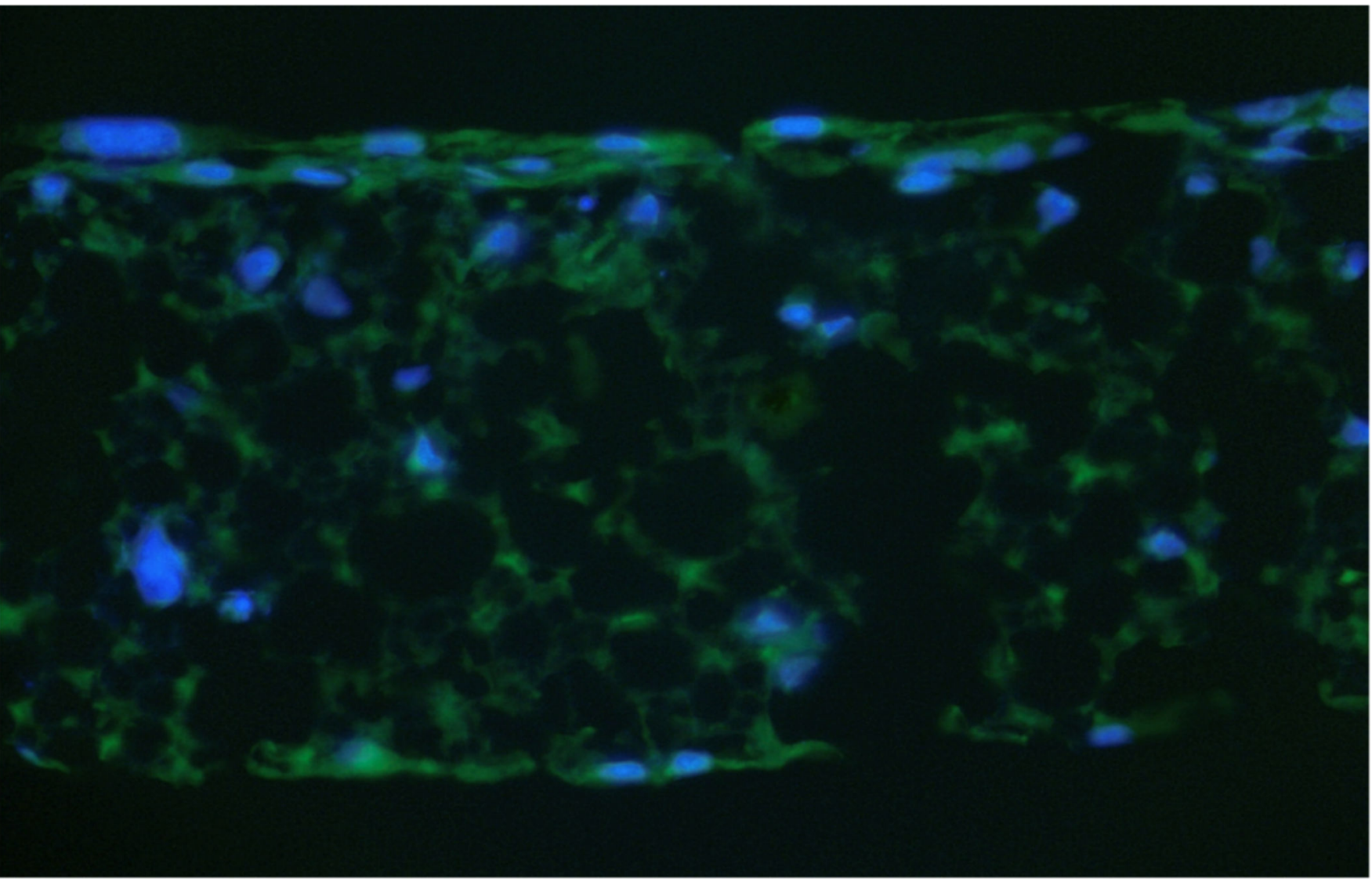

Figure 2.

Calvaria osteocytic cell lines were seeded onto Alvetex polystyrene $(200 \mathrm{~mm})$ scaffolds and grown for 7 days. Scaffolds were fixed in $4 \%$ PFA, cryo-sectioned onto slides at $5 \mathrm{~mm}$, and DAPI stained (blue). Green $=$ GFP positive osteocytes; Blue= DAPI staining. 




Figure 3.

Osteocytes sense mechanical forces (biomechanical stimuli) and transform these stimuli into biological responses (mechano transduction) that results in the secretion of several molecules (Effectors) capable of affecting osteoblasts $(\mathrm{Ob})$ and osteoclast $(\mathrm{Oc})$ functions. Future therapeutics can intervene at three levels, as indicated by the grey arrows. Current therapies acts on the distal cells (ob and oc). 